УДК 621.373 .8

\author{
А.Г. Кузнецов ${ }^{1}$, Д.С. Харенко ${ }^{1}$, В.А. Гонта ${ }^{2}$ \\ ${ }^{1}$ Институт автоматики и электрометрии СО РАН, Новосибирск, Россия \\ ${ }^{2}$ Новосибирский государственный университет, Новосибирск, Россия
}

\title{
УСИЛЕНИЕ ДИССИПАТИВНЫХ СОЛИТОНОВ ТЕЙПЕРНЫМ ВОЛОКОННЫМ УСИЛИТЕЛЕМ
}

\begin{abstract}
Техника генерации сильночирпованных диссипативных солитонов открывает большие возможности для получения мощных лазерных импульсов с короткой длительностью. В данной работе демонстрируется возможность усиления таких импульсов в тейперном волокне с диаметром моды на выходном конце 100 мкм. Сильночирпованные импульсы длительность 10 пс с центральной длиной волны 1040 нм и шириной спектра 13 нм были усилены в полностью волоконной схеме до пиковой мощности 69 кВт и сжаты дифракционными решетками до 800 фс.

Ключевые слова: синхронизация мод, диссипативный солитон, фемтосекундный лазер, тейпер, волокно, иттербиевый усилитель.
\end{abstract}

\author{
A.G. Kuznetsov' ${ }^{1}$, D.S. Kharenko', V.A. Gonta ${ }^{2}$ \\ ${ }^{1}$ Institute for Automation and Electrometry \\ of the Siberian Branch of the Russian Academy of Sciences, \\ Novosibirsk, Russian Federation \\ ${ }^{2}$ Novosibirsk State University, Novosibirsk, Russian Federation \\ AMPLIFICATION OF DISSIPATIVE SOLITONS WITH \\ A TAPER FIBER AMPLIFIER
}

The regime of highly chirped dissipative solitons is a powerful technique for generating high energy short pulses. In this work a possibility of amplification of such pulses with taper active fiber having 100 micron output core diameter is demonstrated. Highly chirped pulses with 10 ps duration, 1040 nm central wavelength and $13 \mathrm{~nm}$ spectral width were amplified in all-fiber scheme up to $69 \mathrm{~kW}$ peak power and compressed to 800 fs duration.

Keywords: mode locking, dissipative soliton, femtosecond laser, taper fiber, ytterbium amplifier.

\section{Введение}

Полностью волоконные лазеры с синхронизацией мод позволяют генерировать короткие импульсы с высоким качеством пучка, обладают хорошей стабильностью и не требуют юстировок оптических элементов. Недавно были продемонстрированы новые схемы резонаторов, исполь- 
зующие как одномодовое волокно, где происходит нелинейное вращение поляризации, так и волокно с сохранением поляризации, в котором формируется диссипативный солитон. Энергия импульса в таких лазеpax ограничена уровнем $\approx 20$ нДж [1], при превышении которого формируется шумовой импульс в другой спектральной области за счет эффекта вынужденного комбинационного рассеяния (ВКР). Использование в резонаторе волокон с большим диаметром моды или LMA-волокна (large mode area) позволяет повысить порог генерации вынужденного комбинационного рассеяния (ВКР) и увеличить энергию импульса до 50 нДж [2], однако и этой энергии недостаточно для многих прикладных задач, таких как микрообработка и фотомодификация материалов. Дальнейшее увеличение выходной мощности лазера возможно при использовании дополнительного волоконного усилителя, например на основе LMA-световода. Так, например, в работе [3] авторы, используя 3 каскада усиления, с помощью LMA-волокон усилили 120 пс импульс до энергии 1,85 мкДж. Дальнейшее увеличение энергии импульса сопровождается сильными нелинейными эффектами. С использованием микроструктурированных оптических волокон (или так называемых PCF - photonic crystal fibers - фотонно-кристаллические волокна) удалось достичь еще больших пиковых мощностей, вплоть до нескольких гигаватт [4]. Однако РCF-усилители не являются полностью волоконными схемами, так как требуют использования объемной оптики для ввода и вывода излучения, кроме того, PCF-волокно должно быть уложено в прямую линию, что делает проблематичным создание компактных устройств. С этой точки зрения перспективным методом усиления является использование тейперного волокна с плавно меняющимся диаметром сердцевины $[5,6]$, что позволяет существенно повысить порог возникновения различных нелинейных эффектов.

Целью данной работы являлось исследование возможности усиления сильночирпованных диссипативных солитонов иттербиевым волоконным усилителем на основе тейперного волокна с последующим их сжатием.

\section{1. Экспериментальная установка}

Схема генератора чирпованных диссипативных солитонов представлена на рис. 1. Лазер выполнен в кольцевой схеме, состоящей из двух функциональных частей: короткого участка из стандартного од- 
номодового волокна, в котором происходит синхронизация мод за счет эффекта нелинейного вращения поляризации, и длинного участка из волокна с сохранением состояния поляризации (РМ - polarization maintaining), в котором формируется диссипативный солитон [1]. Лазер также включает в себя волокно, легированное $\mathrm{Yb}^{3+}$, длиной $20 \mathrm{~cm}$, делитель поляризации (PBS - polarization beam splitter), пигтейлированный волокном, волоконный спектральный фильтр Лио, реализованный привариванием секции РМ-волокна под углом 45, контроллер поляризации и спектрально-селективный ответвитель (WDM - wavelengh division multiplexer), с помощью которого в резонатор заводится излучение накачки мощностью до 300 мВт на длине волны 980 нм от лазерного диода. Направление распространения излучения по волокну задается поляризующим оптическим изолятором с выводами из РМ-волокна.

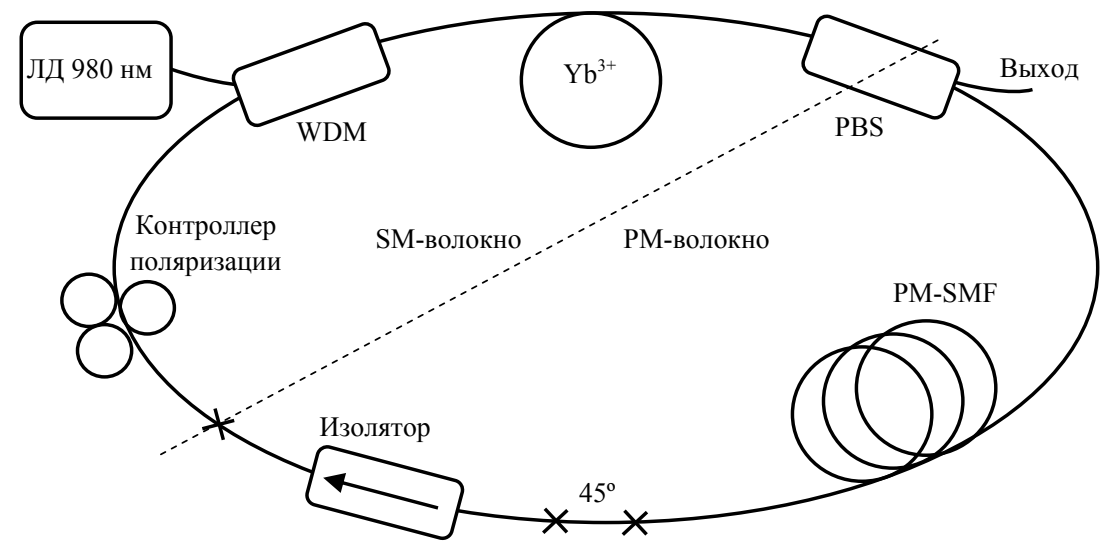

Рис. 1. Схема лазерного источника диссипативных солитонов

Поскольку генератор диссипативных солитонов позволяет генерировать достаточно длинные импульсы с относительно низкой пиковой мощностью, для усиления не требуется использование дополнительного стретчера, и сигнал с выхода задающего осциллятора через изолятор направляется непосредственно на вход усилителя, принципиальная схема которого приведена на рис. 2 [6]. Легированное ионами $\mathrm{Yb}^{3+}$-тейперное волокно представляет собой конусообразный световод с двойной оболочкой длиной 6 м и диаметром сердцевины 15 мкм на его входе и 100 мкм на выходе. С узкой стороны тейпера с помощью волоконного объединителя накачки в световедущую оболочку заводится излучение многомодового лазерного диода с длиной волны гене- 
рации 976 нм и максимальной мощностью 25 Вт. Для накачки усилителя с выходного конца используются два многомодовых диода мощностью по 55 Вт каждый, излучение которых объединяется комбайнером и коллимируется в оболочку. Усиленный полезный сигнал с выхода тейпера направляется дихроичным зеркалом на двухпроходной компрессор с парой дифракционных решеток (1500 штрихов/мм) [7], и сжатый импульс анализируется с помощью системы FROG. Относительно низкая частота повторения задающего генератора позволяет также отказаться от дополнительного прореживания импульсов перед их усилением.

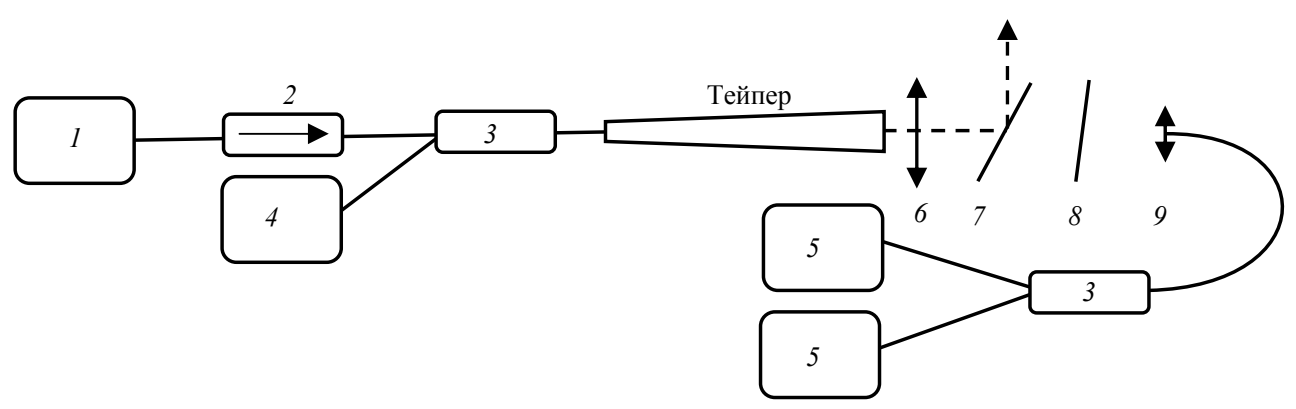

Рис. 2. Схема усиления: 1 - генератор ультракоротких импульсов; 2 - изолятор; 3 - объединитель накачки; 4 - многомодовый диод накачки 976 нм мощностью до 25 Вт; 5 - многомодовый диод накачки мощностью до 55 Вт; 6 - коллиматор;

7 - дихроичное зеркало; 8 - фильтр; 9 - пигтейлированный коллиматор

\section{2. Результаты и обсуждение}

Задающий осциллятор (см. рис. 1) генерировал последовательность сильночирпованных импульсов длительностью 10 пс и с частотой повторения 15 МГц. Средняя мощность лазера составила 20 мВт, центральная длина волны - 1040 нм, а ширина спектра излучения $\approx 13$ нм (рис. 3). Мощность накачки и длина резонатора были выбраны таким образом, чтобы обеспечить максимальную стабильность при отсутствии генерации импульса ВКР.

Далее излучение, проходя через изолятор, заводилось в усиливающее тейперное волокно с двусторонней накачкой. С узкой стороны мощность накачки составляла $25 \mathrm{~B}$, а с широкого конца варьировалась от 0 до 70 Вт. При максимальной мощности накачки с широкого конца (70 Вт) выходная мощность полезного сигнала составила 10,36 Вт, что соответствует пиковой мощности 69 кВт. Его спектр приведен на 
рис. 4. Видно, что при усилении форма спектра в значительной степени искажается, из-за того что рабочая длина волны находится на левом краю профиля усиления иттербиевого волокна. Одновременно с этим из-за большого усиления в спектральной области 1050-1060 нм шумовые компоненты, присутствующие в спектре задающего осциллятора (см. рис. 3), усиливаются и приобретают вид узких множественных паразитных пиков. Очевидно, что для исключения паразитных пиков в выходном спектре требуется более тщательная фильтрация шумов исходного сигнала. На рис. 5 приведена зависимость выходной мощности из усилителя от мощности накачки с широкого конца тейпера.

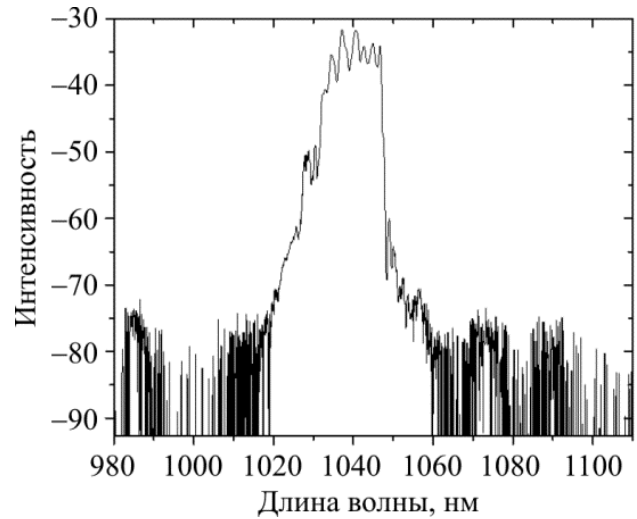

Рис. 3. Спектр излучения задающего осциллятора

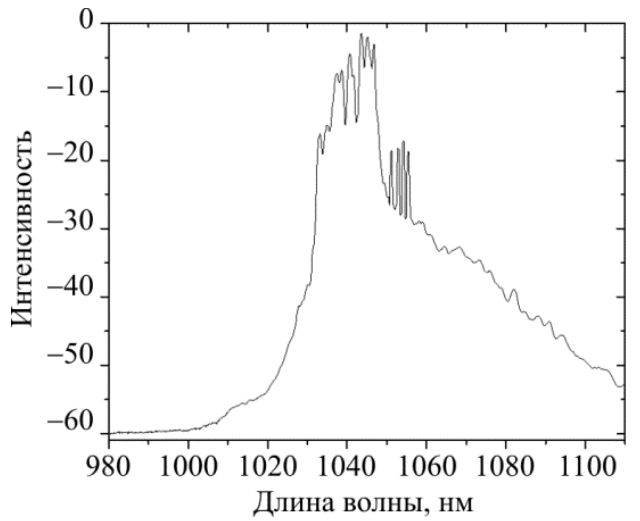

Рис. 4. Спектр усиленного до 10,36 Вт излучения

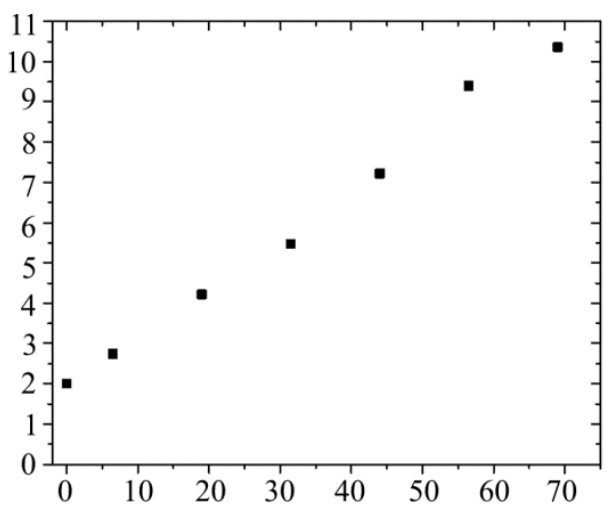

Рис. 5. Зависимость выходной мощности сигнала от мощности накачки с широкого конца тейпера (при включенной накачке 25 Вт с узкого конца) 
Усиленные импульсы сжимались внешним компрессором на дифракционных решетках. Их восстановленный профиль приведен на рис. 6. Длительность сжатого импульса при 8 Вт выходной мощности составила 827 фс, однако с увеличением выходного сигнала до 10 ВТ длительность возрастает до 890 фс, что может быть связано с неравномерным усилением спектрального профиля.
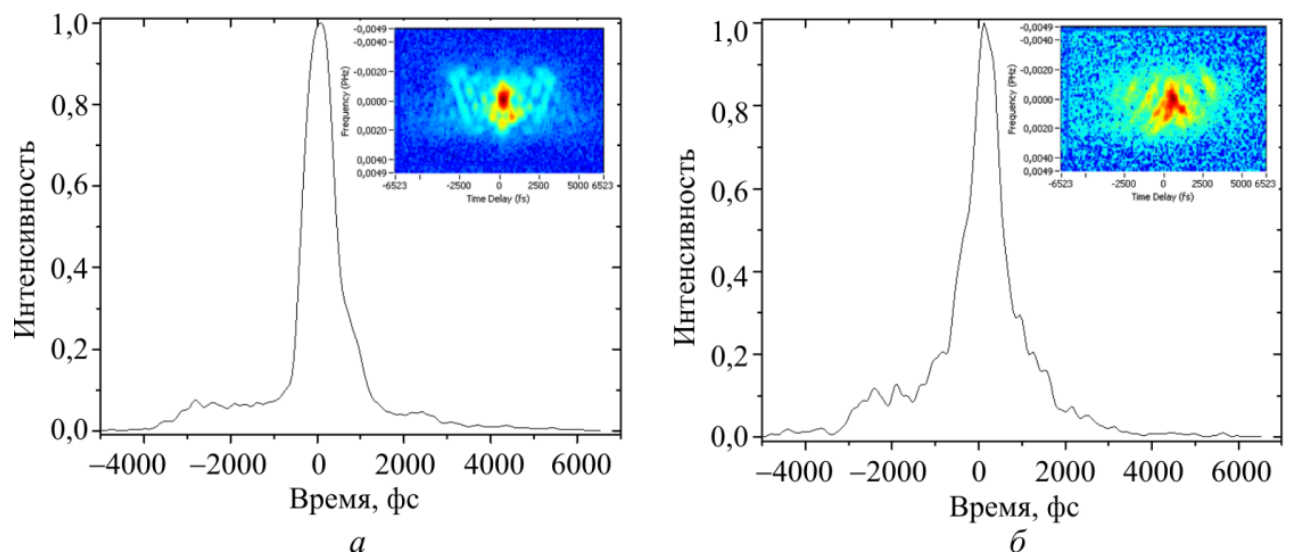

Рис. 6. Профиль сжатых импульсов при выходной мощности 8 Вт ( $a$ ) и 10 Вт $(b)$ с соответствующими FROG-трейсами (вставка)

\section{Заключение}

В работе продемонстрировано усиление тейперным волоконным усилителем сильночирпованных импульсов длительностью 10 пс до пиковой мощности 69 кВт в полностью волоконной схеме. С помощью двухпроходного компрессора с парой дифракционных решеток импульсы были сжаты до $\approx 800$ фс. При усилении сигнала происходят искажение спектра солитонов и возрастание шумов, уже присутствующих на выходе из задающего осциллятора. Поскольку искажение спектра приводит к уменьшению степени сжатия импульса, то перестройка длины волны задающего осциллятора в область 1050-1055 нм могла бы существенно повысить как качество конечного импульса, так и выходную мощность лазерного излучения. Данный лазер может применяться для микрообработки, гравировки и фотомодифицирования материалов.

Работа выполнена при финансовой поддержке междисииплинарного интеграционного проекта СО РАН. 


\section{Список литературы}

1. $20 \mathrm{~nJ} 200$ fs all-fiber highly chirped dissipative soliton oscillator / D.S. Kharenko, E.V Podivilov, A.A. Apolonski, S.A. Babin // Opt. Lett. 2012. - Vol. 37, № 19. - P. 4104-4106.

2. $50 \mathrm{~nJ} 250$ fs all-fibre Raman-free dissipative soliton oscillator / D.S. Kharenko, V.A. Gonta, S.A. Babin // Laser Phys. Lett. - 2016. Vol. 13, № 2. - P. 25107.

3. 1016-nm all fiber picosecond MOPA laser with 50W output / X. Qi, S.-P. Chen, H.-Y. Sun, B.-K. Yang, J. Hou // Opt. Express. - 2016. Vol. 24, № 15. - P. 16874-16883.

4. Fiber chirped-pulse amplification system emitting $38 \mathrm{GW}$ peak power / T. Eidam [et al.] // Opt. Express. - 2011. - Vol. 19, № 1. P. 255.

5. Fibre amplifier based on an ytterbium-doped active tapered fibre for the generation of megawatt peak power ultrashort optical pulses / M.Y. Koptev [et al.] // Quantum Electron. - 2015. - Vol. 45, № 5.

6. Double clad tapered fiber for high power applications / V. Filippov, Y. Chamorovskii, J. Kerttula, K. Golant, M. Pessa, O.G. Okhotnikov // Opt. Express. - 2008. - Vol. 16, № 3. - P. 1929.

7. Feedback-controlled Raman dissipative solitons in a fiber laser / D.S. Kharenko, A.E. Bednyakova, E.V. Podivilov, M.P. Fedoruk, A. Apolonski, S.A. Babin // Opt. Express. - 2015. - Vol. 23, № 2. - P. 1857-1862.

\section{References}

1. Kharenko D.S., Podivilov E.V., Apolonski A.A., Babin S.A. 20 nJ 200 fs all-fiber highly chirped dissipative soliton oscillator. Opt. Lett. 2012, vol. 37, no. 19, pp. 4104-4106.

2. Kharenko D.S., Gonta V.A., Babin S.A. $50 \mathrm{~nJ} 250$ fs all-fibre Raman-free dissipative soliton oscillator. Laser Phys. Lett. 2016, vol. 13, no. 2, p. 25107.

3. Qi X., Chen S.-P., Sun H.-Y., Yang B.-K., Hou J. 1016-nm all fiber picosecond MOPA laser with 50W output. Opt. Express. 2016, vol. 24, no. 15 , pp. 16874-16883.

4. Eidam T. et al. Fiber chirped-pulse amplification system emitting 38 GW peak power. Opt. Express, 2011, vol. 19, no. 1, p. 255. 
5. Koptev M.Y. et al. Fibre amplifier based on an ytterbium-doped active tapered fibre for the generation of megawatt peak power ultrashort optical pulses. Quantum Electron, 2015, vol. 45, no. 5.

6. Filippov V., Chamorovskii Y., Kerttula J., Golant K., Pessa M., Okhotnikov O.G. Double clad tapered fiber for high power applications. Opt. Express, 2008, vol. 16, no. 3, p. 1929.

7. Kharenko D.S., Bednyakova A.E., Podivilov E.V., Fedoruk M.P., Apolonski A., Babin S.A. Feedback-controlled Raman dissipative solitons in a fiber laser. Opt. Express, 2015, vol. 23, no. 2, pp. 1857-1862.

Получено 29.09.2017 